\title{
Off-Pump Coronary Bypass for Patients With Concomitant Malignancy
}

\author{
Feza Nurözler, MD; S. Tolga Kutlu, MD; Güngör Küçük, MD
}

\begin{abstract}
Background The effectiveness and advantages of off-pump coronary artery bypass (OPCAB) for patients with concomitant malignant neoplastic disorders were analyzed.

Methods and Results The study group comprised 368 patients who underwent OPCAB and of them 18 had concomitant malignant neoplastic disease: 14 with solitary malignant disease, 4 with low-grade lymphocytic malignancies. The age of the patients ranged from 49 to 72 years (mean 61). The mean left ventricular ejection fraction was $46.4 \%$ (range: $35-60 \%$ ) and the mean number of grafts was $2.4 \pm 0.9$. The left internal mammary artery was used in 12 patients (75\%). Mean blood loss was $420 \pm 64 \mathrm{ml}$ and the average requirement for blood transfusion was $1.1 \pm 0.2$ units. There was no postoperative infection or in-hospital mortality. The mean length of hospital stay was $6.4 \pm 1.3$ days. Mid-term follow-up was completed $(100 \%)$ and the mean follow-up period was 22.6 \pm 4.1 months. All patients with a solitary malignancy underwent subsequent non-cardiac surgery with a mean interval of $16.4 \pm 3.3$ days. Mid-term survival was $83.4 \%$. Freedom from late cardiac events (angina, myocardial infarction, percutaneous transluminal coronary angioplasty) that required hospital admission was $92.3 \%$.

Conclusion These results support the effectiveness of OPCAB, mostly as a bridge to safe non-cardiac surgery that can be crucial for long-term survival. (Circ J 2006; 70: 1048-1051)
\end{abstract}

Key Words: Coronary artery disease; Malignant neoplastic disease; OPCAB

A

lthough there has been no definitive data about the outcome of patients with a malignant neoplastic disease who underwent coronary bypass, concerns on systemic inflammatory response to cardiopulmonary bypass and its consequences has resulted in an unwillingness in cardiologists to offer coronary artery bypass grafting $(\mathrm{CABG})$ for these patients. However, patients who undergo non-cardiac surgery with known coronary artery disease (CAD), peri-operative cardiac complications have been associated with fatal consequences! If non-surgical revascularization is not suitable, patients should be referred for coronary bypass.

Off-pump coronary artery bypass (OPCAB) surgery through a full median sternotomy has gained renewed interest for multivessel revascularization, particularly for high-risk patients with severe concomitant diseases such as renal, pulmonary, and neurologic dysfunction, 2,3 The relationship between non-cardiac complications and prolonged cardiopulmonary bypass and ischemic times is well documented.,5 Recent studies suggest that overall operative mortality and hospital complications are lower in off-pump operations in high-risk groups?,3,6

Most of the patients with a malignant neoplastic disease are prone to infection and bleeding because of bone marrow suppressive effects of either malignant disease or anticancer treatment. Recently, several reports suggested that patients with concomitant malignant neoplastic disease, the OPCAB surgery has better outcomes and lowered operative mortality because of spared platelet function, less blood

(Received March 27, 2006; revised manuscript received May 22, 2006; accepted June 1, 2006)

Division of Cardiovascular Surgery, Central Hospital, İzmir, Turkey Mailing address: Feza Nurözler, MD, Central Hospital 1644 sok 2/2, Bayrakli, İzmir, Turkey. E-mail: fnurozler@yahoo.com loss, and avoidance of infection?-9 ${ }^{7-9}$ Other than patients having solitary cancer, patients with a low-grade lymphocytic malignancy (including some types of non-Hodgkin's lymphoma and chronic lymphocytic leukemia) can be referred for coronary bypass surgery if a long-term survival is expected.

In this paper our experiences are summarized in the surgical management of CAD in patients who has concomitant malignant neoplastic disease.

\section{Methods}

\section{Patient Population}

A review of cardiac surgery database at our institution from October 2002 to September 2005, showed 368 consecutive patients underwent OPCAB graft (OPCABG) operation by the same surgeon (FN). Eighteen of these patients had concomitant malignant neoplastic disease. Fourteen patients had solitary malignant disease (4 patients

Table 1 Types of Malignant Disease and Stages

\begin{tabular}{lllll}
\hline \hline & $n$ & \multicolumn{3}{c}{ Stage } \\
\cline { 3 - 5 } & & $1 B$ & 2 & $3 A$ \\
\hline Solitary malignancy & 4 & & 3 & 1 \\
$\quad$ Colon cancer & 3 & 1 & 2 & \\
Ovarian cancer & 3 & & & \\
Brain cancer & 2 & & 2 & \\
Gastric cancer & 1 & 1 & & \\
Bladder cancer & 1 & & 1 & \\
Prostate cancer & 3 & & 3 & \\
Heamatologic malignancy & 1 & & 1 & \\
$\quad$ CLL & & & & \\
Non-Hodgkin lymphoma & &
\end{tabular}

CLL, chroic lymphoplastic leukemia. 
Table 2 Preoperative Characteristics

\begin{tabular}{lc}
\hline \hline Age (years) & $61 \pm 1.6$ \\
Gender $(\%$ male) & $13(72)$ \\
Previous MI $(\%)$ & $6(33.3)$ \\
AP $(\%)$ & $14(77)$ \\
Diabetes $(\%)$ & $5(28)$ \\
Hypertension $(\%)$ & $10(55)$ \\
Preoperative EF $(\%)$ & 46.4 \\
EuroSCORE & $3.8 \pm 2.4$ \\
Platelet count $(100 / \mathrm{ml})$ & $215 \pm 45$ \\
Htc $(\%)$ & $33 \pm 8$ \\
\hline
\end{tabular}

MI, myocardial infarction; AP, angina pectoris; EF, ejection fraction; Htc, hematocrit.

Table 3 Severity of Myocardial Ischemia and Indication for CABG

\begin{tabular}{lc}
\hline \hline LMCA lesion & 3 \\
3-vessel disease & 10 \\
2-vessel disease & 3 \\
1 -vessel disease & 2 \\
\hline
\end{tabular}

$C A B G$, coronary artery bypass grafting; LMCA, left main coronary artery.

had colon cancer, 3 patients had ovarian cancer, 3 patients had brain cancer, 2 patients had gastric cancer, 1 patient had bladder cancer, and 1 patient had prostate cancer). The remaining 4 patients were diagnosed with low-grade lymphocytic malignancies (3 patients had chronic lymphoplastic leukemia and 1 patient had non-Hodgkin's lymphoma). Types of malignant disease and stages are summarized in Table 1. The age of patients ranged from 49 to 72 years (mean 61). There were 13 men and 5 women. Six patients had a history of previous myocardial infarction. Mean left ventricular ejection fraction was $46.4 \%$ (range: $35-60 \%$ ). Patients' characteristics are summarized in Table 2. None of those patients had additional severe concomitant diseases other than malignancy. CAD was documented during preoperative work-up for the cancer surgery in 12 patients with solitary malignancy. The remaining 2 patients with solitary malignancy had been followed-up for CAD before malignancy was diagnosed. Any patients with solitary disease did not receive anti-cancer chemothetapy before surgery. The remaining 4 patients with low-grade lymphocytic malignancies had been receiving anti-cancer chemothetapy. Two patients with chronic lymphoplastic leukemia were on fludarabine, whereas the other patient received combination therapy of cyclophosphamide and fludarabine. The patient with non-Hodgkin's lymphoma was on tetrahydropyranil adriamicyn, cyclophosphamide, vincristine, prednisone regimen.

CAD was diagnosed in patients with lymphocytic malignancies when they had been followed up for malignant disease. Severity of myocardial ischemia and indication for CABG are summarized in Table 3. Anticancer chemotherapy was withheld in those patients with low-grade lymphocytic malignancies, 2 weeks before surgery to improve immunity and clot formation, if platelet and/or white blood cell counts were inadequate. Postoperative infections were defined as leukocytosis and/or fever in the presence of a positive culture. Total chest tube drainage during the first $24 \mathrm{~h}$, the incidence of re-exploration, the exposure to blood products, and the incidence of post-operative infection were assessed. Mid-term follow-up was completed by telephone contact either to the referring cardiologist or to the patients.
Table 4 Intraoperative and Postoperative Variables

\begin{tabular}{lc}
\hline \hline No. of distal anastomosis & $2.4 \pm 0.9$ \\
LIMA & $12(75)$ \\
Endarteractomy & $3(16.7)$ \\
Drainage $(m / / 24 h)$ & $420 \pm 64$ \\
Re-exploration $(\%)$ & 0 \\
Packed red blood cells (units/patient) & $1.1 \pm 0.2$ \\
Platelet (units/patient) & 0 \\
Fresh frozen plasma (units/patient) & 0 \\
Mechanical ventilation $>8 h(\%)$ & $1(5.5)$ \\
Length of ICU stay $(h)$ & $16.2 \pm 0.8$ \\
Length of hospital stay (days) & $6.4 \pm 1.3$ \\
Peroperative MI & 0 \\
Postoperative stroke & 0 \\
\hline
\end{tabular}

LIMA, left internal mammary artery; ICU, intensive care unit; MI, myocardial infarction.

\section{Surgical Techniques}

Complete revascularization was aimed in all patients. All coronary artery bypass operations were carried out through a full sternotomy incision. The left internal mammary artery (LIMA) was prepared in all patients other than the patients who received radiotherapy or were a candidate for radiotheraphy for the chest region. Anticoagulation was achieved with heparin $(150 \mathrm{U} / \mathrm{kg})$. No stabilizer was used for stabilization of the left anterior descending artery (LAD), right coronary artery (RCA) diagonal and posterior descending artery (PDA) branches. Exposure of LAD and diagonal branch is achieved by placing a sponge under the heart and a deep pericardial traction suture to elevate and slightly rotate the heart rightward. Exposure of RCA and PDA branches is achieved by rotating the heart leftward with rightward traction of an unfold gauze placed at the base of the heart. Stabilization of the heart has been accomplished by using 2 stay sutures (3-0 braided coated polyester) placed proximally and distally to the area planned for arteriotomy. Exposure to circumflex system and posterolateral branch of the RCA was obtained with traction of 2 deep pericardial traction sutures placed near the left upper pulmonary veins and to the left of the inferior vena cava, thereby achieving vertical displacement of the apex of the heart. To obtain further exposure of the target arteries on the lateral and inferior aspect of the heart, the patients were also slightly rotated to the right decubitus Trendelenburg position. Right pleura opened widely and the right side of the sternum was lifted up by a rolled compress placed underneath of the right arm of the sternum retractor. Then the heart was lifted up and rotated rightward and placed in the right hemithorax. Gauze was placed between the heart and right side of the sternum. Octopus I stabilizer (Medtronic, Minneapolis, MN, USA) was seldom applied to the arteries of circumflex system and postero-lateral branch of the RCA when proximal part of the arteries were chosen for anastomosis. Two stay sutures (3-0 braided coated polyester) were placed proximal and distal to the area planned for arteriotomy. Inferior vena cava was also freed from the peri-cardium and diaphragm as much as possible to prevent kinking of the vena cava so that the heart could tolerate vertical displacement. Proximal anastomosis was performed under aortic side-biting clamp.

\section{Results}

The mean number of CABG grafts per patient was $2.4 \pm 0.9$. The number of $\mathrm{CABG}$ grafts performed was 
single in 2 patients, double in 7 patients, triple in 6 patients, and quadruple in 3 patients. LIMA was used in 12 patients (75\%). Three patients required coronary endarterectomy to achieve an adequate run-off. Indication to perform coronary endarterectomy was made intraoperatively after arteriotomy showed either an occluded lumen with no graftable vessel or if a 1-mm coronary probe could not be passed down the target vessel. None of the patients was converted to on-pump coronary bypass because of hemodynamic deterioration. Total amount of postoperative blood loss was $300-650 \mathrm{ml}$, with a mean of $420 \pm 64 \mathrm{ml}$. Average requirement for blood transfusion was $1.1 \pm 0.2$ units. Platelet or fresh frozen plasma transfusion were not required. Mediastinal re-exploration was not required in patients. No infection was documented during the postoperative course. There was no in-hospital mortality. Following uneventful postoperative course, the patients were discharged at a mean of $6.4 \pm 1.3$ days after surgery.

\section{Mid-Term Morbidity and Mortality}

Mid-term follow-up was completed in all patients. The mean follow-up period was $22.6 \pm 4.1$ months. All patients with a solitary malignancy underwent a non-cardiac surgery after coronary bypass with a mean interval of $16.4 \pm 3.3$ days. All patients survived non-cardiac surgery. Three patients died in the mid-term period. Mid-term survival was $83.4 \%$. None of the deaths was cardiac in origin and were caused by complications of malignant disease. Freedom from late cardiac events (angina, myocardial infarction, percutaneous transluminal coronary angioplasty (PTCA)) that required hospital admission was $92.3 \%$. Among the 13 patients in whom late follow-up was completed, 1 patient developed recurrent myocardial infarction in a revascularized territory (the RCA).

\section{Discussion}

In the management of CAD in patients with known concomitant malignant neoplastic disease, coronary bypass surgery is seldom recommended. Concerns about systemic inflammatory response to cardiopulmonary bypass and its consequences, such as interstitial fluid accumulation and tissue edema formation, impaired immune response, and decreased platelet number and function, ${ }^{4,5}$ are factors that make these patients not referred to cardiac surgery, unless a non-cardiac surgery for cancer is necessary with an expectation of long-term survival. Although there has been no definitive data about the outcome of patients with a malignant neoplastic disease who underwent coronary bypass, cardiologists are unwilling to offer CABG for those patients. Possible systemic tumor dissemination because of alteration of the immune system or direct vascular dissemination is another concern during resections performed with $\mathrm{CPB} !^{10}$ Avoidance of CPB is beneficial for the treatment of malignant neoplastic diseases.

However, OPCAB surgery through a full median sternotomy has gained renewed interest for multivessel revascularization and widened the possibilities for surgical treatment of ischemic heart disease in patients with various comorbities for which CPB is contraindicated. In the surgical management of CAD, many surgeons have adopted complete revascularization. Complete revascularization can be sensed as hard to deal with especially in patients with multiple vessel CAD. However, with the development of various surgical techniques, anesthetic management and technologies, almost all coronary arteries can be bypassed off-pump. Recent studies suggest that overall operative mortality and hospital complications are lower in off-pump operations in high-risk groups $2,3,6$ Most of the patients with a malignant neoplastic disease are prone to infection and bleeding because of bone marrow suppressive effects of either malignant disease or anticancer treatment. Recently, several reports suggested that patients with concomitant malignant neoplastic disease, OPCAB surgery has better outcomes and lowered operative mortality because of spared platelet function, less blood loss, and avoidance of infection? ${ }^{\text {-9 }}$

Other than the abovementioned points, a unique situation exists in patients with a brain tumor. Application offpump bypass to these patients is of paramount importance because cardiopulmonary bypass causes tissue edema and consequently increased compression effect of the tumor. This might result in mortality or persistent sequel.

Considering the shorter recovery time than conventional bypass, off-pump bypass offers another very important advantage, because most of the patients need to undergo a non-cardiac surgery soon after coronary bypass.

The eradication of CPB from coronary bypass operation in patients with malignancy is of paramount importance because CABG plays the role of ensuring the safety of subsequent treatment of the neoplastic disease. In patients who undergo non-cardiac surgery with known $\mathrm{CAD}$, peri-operative cardiac complication has been associated with fatal consequences! If non-surgical revascularization is suitable, it should be preferred in this circumstance. Nevertheless, CABG is advantageous over PTCA. CABG can provide consistent and more durable revascularization than PTCA and furthermore the patients do not need to take anticoagulant or powerful anti-aggregant treatment that are often necessary after PTCA and which might be associated with bleeding complication on subsequent treatment of neoplastic disorder.

Evolution of off-pump CABG and improved results with high-risk patient have resulted in more patients with a malignant neoplastic disorder being referred for CABG. In this small series of patients, OPCABG was carried out with good early and mid-term results. These results support the effectiveness of OPCAB mostly as a bridge to safe noncardiac surgery that can be crucial for long-term survival in those patients. Further studies with a long-term follow-up are needed to support and improve the results of OPCAB in patients who has concomitant malignant disease.

\section{Study Limitation}

The limitation of this study is its retrospective design and lack of a control group of conventional coronary by-pass. Furthermore, there is a lack of late angiographic follow-up to assess graft patency. Long-term follow-up is required to support this finding.

\section{References}

1. Ashton CM, Petersen NJ, Wray NP, Kiefe CI, Dunn JK, Wu L, et al. The incidence of perioperative myocardial infarction in man undergoing noncardiac surgery. Ann Intern Med 1993; 118: 504-510.

2. Akiyama K, Ogasawara K, Inoue T, Shindou S, Okumura H, Negishi $\mathrm{N}$, et al. Myocardial revascularization without cardiopulmonary bypass in patients with operative risk factors. Ann Thorac Cardiovasc Surg 1999; 5: 31-35.

3. Nakamura Y, Nakano K, Nakatani H, Gomi A, Sato A, Sugimoto K. Hospital and mid-term outcomes in elderly patients under-going offpump coronary artery bypass grafting comparison with younger 
patients. Circ J 2004; 68: 1184-1188.

4. Kirklin JK,Westaby S, Blackstone EH. Complement and the damaging effects of cardiopulmonary bypass. J Thorac Cardiovasc Surg 1983; 86: $845-857$.

5. Butler J, Rocker GM, Westaby S. Inflammatory response to cardiopulmonary bypass. Ann Thorac Surg 1993; 55: 552-559.

6. Ishida M, Junjiro J, Tagusari O, Bando K, Niwaya K, Nakajima H, et al. Perioperative advantages of off-pump coronary artery bypass grafting. Circ J 2002; 66: 795-799.

7. Hirose H, Amano A, Yoshida S, Nagao T, Sunami H, Takahashi A, et al. Coronary artery bypass grafting in patient with malignant neo- plasm. Efficacy of coronary artery bypass grafting on beating heart. Jpn J Thorac Cardiovasc Surg 2000; 48: 96-100.

8. Ghosh P, Carroll I, Kanhere A, Ng B, Larbalestier R, Edwards M. Cardiac operations in patients with low-grade small lymphocytic malignancies. J Thorac Cardiovasc Surg 1999; 118: 1033-1037.

9. Ochi M, Yamada K, Fujii M, Ohkubo N, Ogasawara H, Tanaka S. Role of off-pump coronary artery bypass grafting in patients with malignant neoplastic disease. Jpn Circ J 2000; 64: 13-17.

10. Peters RM, Swain JA. Management of the patient with emphysema, coronary artery disease, and lung cancer. Am J Surg 1982; 143: 701 705 . 